\title{
Gynaecologists estimate and experience laparoscopic hysterectomy as more difficult compared with abdominal hysterectomy
}

\author{
Theodoor E. Nieboer • Marc E. A. Spaanderman • \\ Marlies Y. Bongers • Mark E. Vierhout • \\ Kirsten B. Kluivers
}

Received: 30 December 2009 / Accepted: 12 May 2010 / Published online: 28 May 2010

(C) Springer-Verlag 2010

\begin{abstract}
The level of difficulty of various types of hysterectomy differs and may influence the choice of either approach. When surgeons consider one specific approach to hysterectomy as more difficult, they may be reluctant to perform this type of hysterectomy. The main objective of this study was to investigate the potential different levels of difficulty for laparoscopic and abdominal hysterectomy. Furthermore, the accuracy of estimating the level of difficulty was examined. In a randomized controlled trial between laparoscopic hysterectomy ( $\mathrm{LH})$ and abdominal hysterectomy $(\mathrm{AH})$, gynaecologists were asked to record the preoperatively estimated and postoperatively experienced level of difficulty on a Visual Analogue Scale (VAS). Differences between LH and $\mathrm{AH}$ were examined and the correlation between the estimated uterine weight on bimanual palpation and the actual uterine weight was calculated. A difference on the VAS of three points or more $(\triangle \mathrm{VAS} \geq 3)$ was considered clinically relevant. In 72 out of 76 cases, both VAS scores were recorded. LH was estimated and experienced as significantly more difficult as compared with AH. In 13 (18\%) cases, $\triangle$ VAS was $\geq 3$, equally distributed between LH $(n=6)$ and $\mathrm{AH}(n=7)$. Eleven of these 13 cases had a positive $\Delta \mathrm{VAS} \geq 3$, meaning that surgery was experienced as more difficult than it
\end{abstract}

We report no funding or other sources of financial support. All authors contributed to the creation of this manuscript.

T. E. Nieboer $(\bowtie) \cdot$ M. E. A. Spaanderman $\cdot$ M. E. Vierhout $\cdot$

K. B. Kluivers

Department of Obstetrics and Gynecology,

Radboud University Nijmegen Medical Centre,

P/O Box 9101, 6500 HB Nijmegen, The Netherlands

e-mail: b.nieboer@obgyn.umcn.nl

\section{Y. Bongers}

Department of Obstetrics and Gynecology,

Maxima Medical Centre Veldhoven,

Veldhoven, The Netherlands was estimated. Surgeon's estimation of uterine size correlated well with the actual uterine weight. LH is considered as more difficult than $\mathrm{AH}$, which might be a reason for its slow implementation. In a large proportion of cases, gynaecologists seem to be able to estimate the level of difficulty of hysterectomy accurately.

Keywords Randomized trial - Laparoscopic hysterectomy . Abdominal hysterectomy Level of difficulty

\section{Introduction}

Hysterectomy is the most frequently performed major gynaecological surgical procedure [1]. Vaginal hysterectomy (VH) is considered the least invasive approach [2]. However, if $\mathrm{VH}$ hysterectomy is not possible, laparoscopic hysterectomy (LH) may avoid the need of a laparotomy in a large proportion of cases. Since the first description of LH by Reich et al. in 1989 [3], this procedure has been implemented worldwide. In the Netherlands, however, the implementation of laparoscopic hysterectomy seems to develop at a slow pace. Kolkman et al. [4] reported that laparoscopic-assisted vaginal hysterectomy (LAVH) was performed in only $58 \%$ of hospitals and only $4 \%$ of hysterectomies were LAVHs. Lack of laparoscopic training and experience during residency could be a factor of importance on this matter [5]. With less experience, gynaecologists who plan hysterectomies may judge LH as too difficult and therefore may have a tendency to perform an abdominal hysterectomy when vaginal hysterectomy is not possible.

Several factors may influence the estimated level of difficulty of hysterectomy: uterine size on bimanual palpation, patients' weight and BMI, previous abdominal surgery and surgeon's experience with the planned approach to 
hysterectomy. Furthermore, the estimated level of difficulty itself may have an impact on the scheduled amount of time for surgery. In order to optimize the logistic patterns of operating theatre, next to accurate day case management, it is mandatory that surgeons planning hysterectomy make adequate estimations of the difficulty of surgery [6-8].

Until now, it is not known whether surgeons experience abdominal and laparoscopic hysterectomy as equally difficult. The main objective of this study was to assess whether there was a difference in estimated and experienced difficulty between abdominal and laparoscopic hysterectomy.

\section{Materials and methods}

Between August 2002 and January 2005, a randomized controlled trial was conducted, in which women were randomized to either abdominal or laparoscopic hysterectomy. For benign indications, a vaginal hysterectomy was performed when the size of the uterus did not exceed 12 weeks' gestation with the cervix descending until at least halfway the vagina under cervical traction with a tenaculum forceps. In cases in which these conditions for vaginal hysterectomy were not met and the size of the uterus did not exceed 18 weeks' gestation, patients were eligible for the study. Exclusion criteria were suspicion of malignancy other than endometrial carcinoma, a previous lower midline incision, the need for simultaneous interventions like prolapse repair and inability to speak Dutch. Written informed consent was required. We obtained approval for the study from the hospital's Medical Ethical Committee.

We recorded body mass index (BMI), uterine size on bimanual palpation, the surgeons' number of previously performed hysterectomies, operation time, conversions in case of LHs and pre- and postoperative complication rate. The laparoscopic procedures were all intentionally total laparoscopic hysterectomies (TLH). The surgical technique has been described in detail before [9]. The abdominal hysterectomies were performed through a transverse incision using the standard extrafascial technique. The surgeon's number of previously performed hysterectomies was used as a measure of surgical experience. Preoperatively, all gynaecologists were asked to rate the estimated level of difficulty on a 1-10 visual analogue scale (VAS) with the patient under anaesthesia. Postoperatively, the same was done for the experienced level of difficulty. Higher scores denote a higher difficulty level. A difference of three or more points between the estimated and experienced score $(\triangle \mathrm{VAS} \geq 3)$ was considered as clinically relevant. Furthermore, we analysed factors that could possibly be related to these cases of $\triangle \mathrm{VAS} \geq 3$ and factors related to cases in which a complication occurred. As a measure of the surgeon's ability to perform accurate estimations, we analysed the correlation between the estimated uterine size on bimanual palpation and the actual uterine weight on pathological examination.

Statistical analysis was performed using Statistical Package for the Social Sciences (SPSS) 16.0. Data are detailed as mean and standard deviation or median and interquartile range and analysed by Mann-Whitney test or chi-square test whenever applicable. Spearman's rho analysis was used to determine potential correlations. $P$-values below .05 were considered statistically significant.

\section{Results}

Out of 76 patients randomised, both pre- and postoperative VAS scores were recorded by gynaecologists in 72 patients (95\%). Table 1 shows the baseline patient characteristics and surgical parameters. As expected, LH required a longer surgery time and was accompanied with less blood loss. Compared with $\mathrm{AH}, \mathrm{LH}$ was estimated and experienced as more difficult. A subgroup analysis revealed that surgical experience (number of previous hysterectomies) was inversely related to the estimated level of difficulty in $\mathrm{AH}$ (Spearman's rho $-0.365 ; p=0.031$ ), but not in LH.

In 13 patients (18\%), there was a $\triangle \mathrm{VAS} \geq 3$ (surgery was estimated as significantly less or more difficult compared to the actual experienced difficulty; see Fig. 1). These 13 cases were equally divided between the laparoscopic $(n=6)$ and abdominal $(n=7)$ approach. In 11 out of $13(85 \%)$ cases, there was a positive $\triangle \mathrm{VAS} \geq 3$ (surgery was experienced as more difficult than it was estimated). The two patients in whom experienced difficulty was significantly lower compared to estimated difficulty were both AHs. Conversion from laparoscopy to laparotomy did not occur in these 11 patients.

Table 1 Baseline patients' characteristics and surgical parameters

\begin{tabular}{lrrr}
\hline & AH $(n=35)$ & LH $(n=37)$ & $p$ \\
\hline Age (years) & $48.1 \pm 9.5$ & $50.0 \pm 9.2$ & 0.208 \\
Parity & $1.5 \pm 1.3$ & $1.9 \pm 1.2$ & 0.137 \\
BMI (kg/m $\left.{ }^{2}\right)$ & $26.4 \pm 3.9$ & $26.7 \pm 5.8$ & 0.774 \\
Uterine size (weeks) $^{\text {Estimated difficulty }}{ }^{\mathrm{a}}$ & $9.2 \pm 4.2$ & $9.7 \pm 5.2$ & 0.875 \\
Experienced difficulty $^{\mathrm{a}}$ & $3.0 \pm 1.6$ & $4.3 \pm 2.4$ & 0.023 \\
Surgery time (min) $_{\text {Blood loss (ml) }}$ & $82 \pm 32.2$ & $4.8 \pm 2.5$ & 0.018 \\
\hline
\end{tabular}

Data are presented as mean \pm standard deviation

$A H$ abdominal hysterectomy, $L H$ laparoscopic hysterectomy

${ }^{\mathrm{a}}$ Estimated and experienced difficulty rated on a $0-10$ Visual Analogue Scale, where a higher score denotes a higher difficulty level 


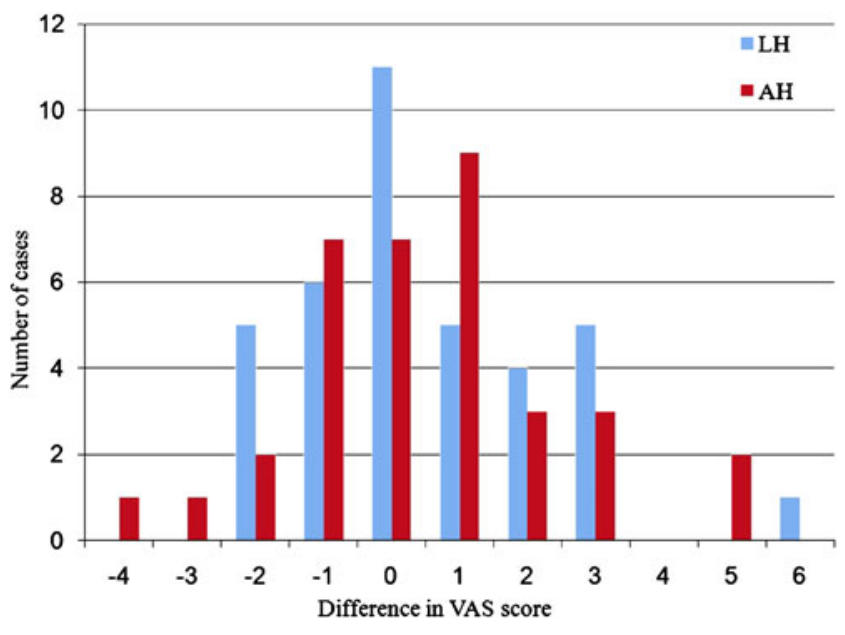

Fig. 1 Difference between estimated and experienced difficulty level in laparoscopic $(L H)$ and abdominal hysterectomy $(A H)$. VAS, Visual Analogue Scale. A positive difference indicates that surgery was experienced as more difficult than it was estimated

Table 2 shows the cases of positive $\triangle \mathrm{VAS} \geq 3$ in relation to BMI, operation time, intubation time, total time in operating room, blood loss, uterine weight, surgeons' number of previously performed hysterectomies and complication rate. Surgery time, intubation time and total time in operating room were longer in more difficult cases (with positive $\triangle \mathrm{VAS} \geq 3$ ), although $95 \%$ confidence intervals crossed zero.

Figure 2 shows the correlation between the estimated uterine size on bimanual palpation and the actual uterine weight on pathological examination. With Spearman's rho= $0.702(p<0.001)$, the estimated size of the uterus correlated well with the actual weight.

In nine patients (12.5\%), a complication occurred (four conversions and one bladder lesion in LH; two bleedings $>1,000 \mathrm{cc}$, one severe allergic reaction and one unintentionally subtotal hysterectomy in $\mathrm{AH}$ ). In these cases, BMI and both the estimated and experienced difficulty was higher (Table 3).

\section{Discussion}

To our knowledge, this is the first study in which the estimated and the experienced level of difficulty of hysterectomy, respectively, have been compared. We have found that laparoscopic hysterectomy is estimated and experienced as more difficult compared to abdominal hysterectomy. We also observed that the level of difficulty in about one out of five operations is not correctly estimated.

The subjective higher level of difficulty of LH versus AH may have a substantial role in the slow implementation of laparoscopic hysterectomy in The Netherlands. As mentioned by Kolkman et al. in 2002, laparoscopicassisted vaginal hysterectomy was performed in only $58 \%$ of hospitals and only $4 \%$ of hysterectomies were LAVHs [4]. Total laparoscopic hysterectomy was not reported to be performed. More laparoscopic training and experience during residency may partly melt away this subjective higher level of difficulty. Many initiatives have already been taken to enhance the practise hours on virtual reality and box trainers. Results from a Dutch nationwide study on laparoscopic hysterectomy will soon reveal its current implementation.

More surgical experience with $\mathrm{AH}$ seems to be associated with a lower estimated level of difficulty. This association was not observed in LH, which might be due to the fact that all LHs were performed by experienced laparoscopic surgeons, whereas AHs were also performed by residents in training. Many authors have reported on the

Table 2 Factors possibly related to $\triangle$ VAS

\begin{tabular}{llll}
\hline & $\Delta$ VAS $<3(n=59)$ & $\Delta$ VAS $\geq 3(n=11)^{\mathrm{a}}$ & Mean difference (95\% CI) \\
\hline BMI $\left(\mathrm{kg} / \mathrm{m}^{2}\right)$ & $26.5 \pm 5.2$ & $26.7 \pm 4.4$ & NA \\
Time of surgery (min) & $99 \pm 37$ & $119 \pm 35$ & $20(-5$ to 44$)$ \\
Time of intubation (min) & $120 \pm 40$ & $142 \pm 34$ & $22(-4$ to 47$)$ \\
Time on OR (min) & $129 \pm 43$ & $150 \pm 31$ & $21(-2$ to 44$)$ \\
Blood loss (cc) & $288 \pm 269$ & $382 \pm 227$ & $94(-70$ to 257$)$ \\
Uterine weight (gram) & $220 \pm 182$ & $215 \pm 154$ & NA \\
Surgeon's experience & $30(9-100)$ & $30(13-100)$ & NA \\
Complications $(\%)^{\mathrm{c}}$ & $8(13.6)$ & $1(9.1)$ & NA \\
\hline
\end{tabular}

Data presented as mean \pm standard deviation. Surgeon's experience refers to the number of previously performed hysterectomies

${ }^{a}$ Only 11 cases with a positive $\triangle \mathrm{VAS} \geq 3$ were used for this analysis (cases where experienced difficulty was higher than estimated difficulty)

${ }^{\mathrm{b}} 95 \%$ confidence intervals of the mean difference (independent sample $t$-test)

${ }^{\mathrm{c}}$ Data presented as median (range)

${ }^{\mathrm{d}}$ Data presented as number (percentage) 
Fig. 2 Scatter box plot showing the correlation between estimated uterine size and actual uterine weight. Spearman's rho $=0.702$ $(p<0.001)$. Line was assigned using curve fitting in SPSS 16.0

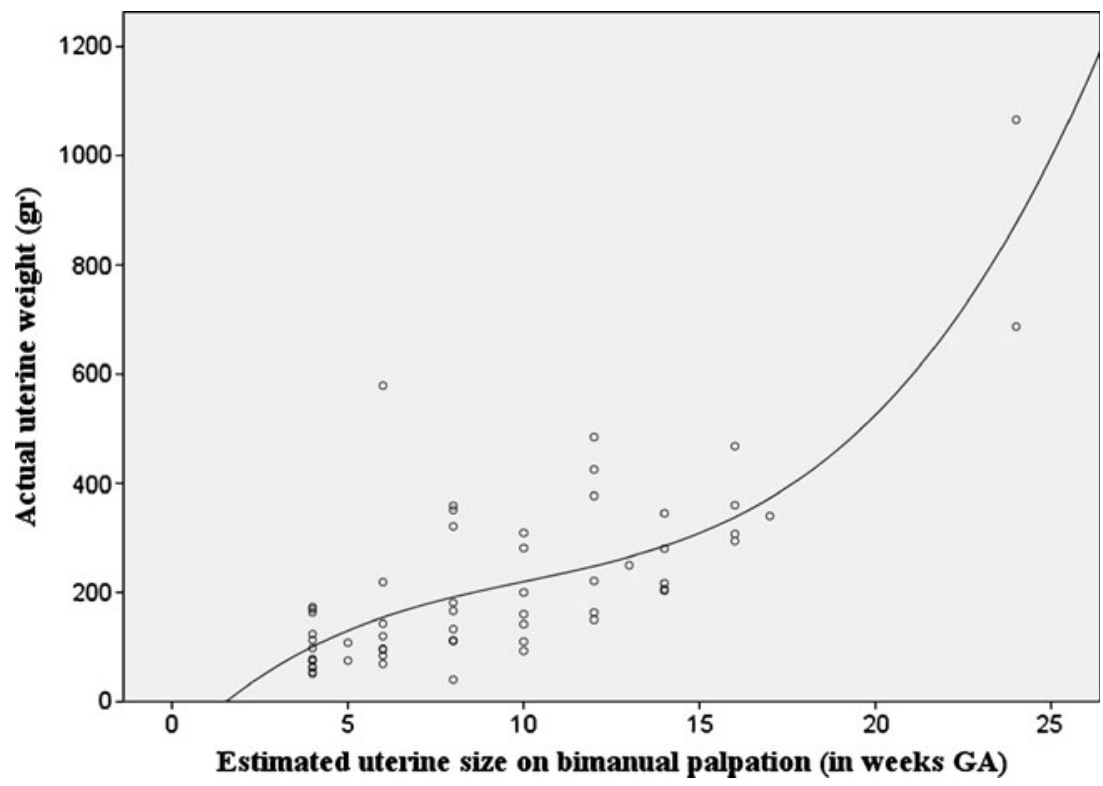

learning curve for LH [10-13] and, in general, concluded that, with increasing experience, operation time and complication rate decreased. In literature, data on the learning curve for AH are scarce. Yaegashi et al. [14] concluded that from $25 \mathrm{AHs}$ and onwards blood loss is reduced and surgical time reduces from $75 \mathrm{AHs}$ and onwards. Leminen et al. [15] stated that an increased experience had no effect on complication rates in $\mathrm{AHs}$, but a decrease of $44 \%$ was seen in LHs.

Previous studies have reported on factors that may influence operating room planning [6-8, 16, 17]. Although adequate estimation of surgery time for a specific procedure is frequently mentioned as an important factor, refinement of OR allocations a few months before the day of surgery as well as management decisions on the day of surgery appear to be more relevant in preventing over- or underutilization of operating rooms $[7,16]$. In a large observational study, average underestimation of the required surgery time by surgeons and schedulers was $22 \mathrm{~min}$ for each $8 \mathrm{~h}$ of OR time [8]. In our study, patients in which surgery was estimated less difficult than it was experienced (positive $\triangle \mathrm{VAS} \geq 3$ ) required on average $21 \mathrm{~min}$ longer $\mathrm{OR}$ time. However, as confidence intervals just crossed zero and this short overutilization of an operating room is likely to be levelled by underutilization of other ORs and up-to-date day case management, no strict conclusions can be drawn from this result. Furthermore, as our study concerned a randomized trial in which the scheduler was not necessarily the surgeon, reticence on extrapolating these findings to every day practise seems argumentative.

Interestingly, we found higher estimated difficulty levels in cases where a complication occurred. This might be an indication for surgeons being capable of predicting the risk of complications. Others have studied the possibility of predicting the risk of complications in gynaecologic (laparoscopic) surgery. Mirashemi et al. found that, besides the type of laparoscopic surgery, increasing age was the most important predictor of complications [18]. In our study, age was not related to the occurrence of complications (data not shown). Myers et al. developed a model to predict the medical and the surgical complications of hysterectomy [19]; they incorporated demographic, diagnostic and procedural data. However, they concluded that the use of routinely collected administrative data for risk adjustment of hysterectomy complication rates cannot be recommended. Dean et al. concluded that the mean length of hospital stay was longer and complication rate was higher in patients with two or more co-morbidities, age above 60 and higher American Society of Anesthesiologists classification score [20]. Several other groups tried to predict which patients were at risk for postoperative

Table 3 Factors possibly related to surgical complications

\begin{tabular}{llll}
\hline & $\begin{array}{l}\text { Complication } \\
(n=9)\end{array}$ & $\begin{array}{l}\text { No complication } \\
(n=63)\end{array}$ & $p$ \\
\hline $\begin{array}{l}\text { Preoperative VAS } \\
\text { score }\end{array}$ & $6.9 \pm 2.8$ & $3.2 \pm 1.6$ & $<0.01$ \\
$\begin{array}{l}\text { Postoperative VAS } \\
\text { score }\end{array}$ & $7.3 \pm 2.7$ & $3.6 \pm 2.1$ & $<0.01$ \\
$\begin{array}{l}\text { BMI }\left(\mathrm{kg} / \mathrm{m}^{2}\right) \\
\text { Size of uterus (gr) }\end{array}$ & $30.4 \pm 8.4$ & $26.0 \pm 4.1$ & 0.014 \\
Surgeon's experience & $316 \pm 368$ & $205 \pm 123$ & 0.812 \\
\hline
\end{tabular}

Data presented as mean \pm standard deviation. Surgeon's experience refers to a surgeon's number of previously performed hysterectomies VAS 1-10 Visual Analogue Scale, where higher scores denote a higher difficulty level.

${ }^{a}$ Data presented as median (range) 
infection in $\mathrm{AH}[21,22]$; they concluded that bacterial vaginosis and trichomoniasis vaginitis are risk factors for the development of posthysterectomy cuff cellulitis. All mentioned factors can be used to coordinate pre-, intra- and postoperative care.

There are some possible limitations of our study. Firstly, the preoperative estimation of level of difficulty was performed with the patient under anaesthesia. This may have led to a slightly different estimation as compared to judgement in the outpatient setting. Secondly, the scheduler was not necessarily the surgeon who performed the hysterectomy and multiple surgeons scheduled and performed the hysterectomies. Furthermore, in cases with high estimated and experienced VAS scores, the reason for these high scores was not reported by surgeons. Finally, the difference in VAS score of three units is a chosen cutoff point.

In conclusion, we have found that laparoscopic hysterectomy was estimated and experienced as more difficult compared to abdominal hysterectomy. This may be a matter of importance in the slow implementation of LH in The Netherlands. Further studies on the effect of enhancing laparoscopic training and experience are needed.

\section{References}

1. Brolmann HA, Vervest HA, Heineman MJ (2001) Declining trend in major gynaecological surgery in The Netherlands during 19911998. Is there an impact on surgical skills and innovative ability? BJOG 108:743-748

2. Nieboer TE, Johnson N, Lethaby A, Tavender E, Curr E, Garry R, van Voorst S, Mol BW, Kluivers KB (2009) Surgical approach to hysterectomy for benign gynaecological disease. Cochrane Database Syst Rev 8(3):CD003677

3. Reich H, DeCaprio J, McGlynn F (1989) Laparoscopic hysterectomy. J Gynecol Surg 5:213-216

4. Kolkman W, Trimbos-Kemper TC, Jansen FW (2007) Operative laparoscopy in The Netherlands: diffusion and acceptance. Eur J Obstet Gynecol Reprod Biol 130:245-248

5. Kolkman W, Wolterbeek R, Jansen FW (2005) Gynecological laparoscopy in residency training program: Dutch perspectives. Surg Endosc 19:1498-1502

6. Wright IH, Kooperberg C, Bonar BA, Bashein G (1996) Statistical modeling to predict elective surgery time. Comparison with a computer scheduling system and surgeon-provided estimates. Anesthesiology 85:1235-1245

7. Dexter F, Epstein RH, Traub RD, Xiao Y (2004) Making management decisions on the day of surgery based on operating room efficiency and patient waiting times. Anesthesiology 101:1444 1453

8. Dexter F, Macario A, Ledolter J (2007) Identification of systematic underestimation (bias) of case durations during case scheduling would not markedly reduce overutilized operating room time. J Clin Anesth 19:198-203

9. Kluivers KB, Hendriks JC, Mol BW, Bongers MY, Bremer GL, De Vet HC, Vierhout ME, Brolmann HA (2007) Quality of life and surgical outcome after total laparoscopic hysterectomy versus total abdominal hysterectomy for benign disease: a randomized, controlled trial. J Minim Invasive Gynecol 14:145-152

10. Brummer TH, Seppala TT, Harkki PS (2008) National learning curve for laparoscopic hysterectomy and trends in hysterectomy in Finland 2000-2005. Hum Reprod 23:840-845

11. Perino A, Cucinella G, Venezia R, Castelli A, Cittadini E (1999) Total laparoscopic hysterectomy versus total abdominal hysterectomy: an assessment of the learning curve in a prospective randomized study. Hum Reprod 14:2996-2999

12. Wattiez A, Soriano D, Cohen SB, Nervo P, Canis M, Botchorisvili R, Mage G, Pouly JL, Mille P, Bruhat MA (2002) The learning curve of total laparoscopic hysterectomy: comparative analysis of 1647 cases. J Am Assoc Gynecol Laparosc 9:339

13. Harkki-Siren P, Sjoberg J (1995) Evaluation and the learning curve of the first one hundred laparoscopic hysterectomies. Acta Obstet Gynecol Scand 74:638-641

14. Yaegashi N, Kuramoto M, Nakayama C, Nakano M, Yajima A (1996) Resident gynecologists and total hysterectomy. Tohoku J Exp Med 178:299-306

15. Leminen A (2000) Comparison between personal learning curves for abdominal and laparoscopic hysterectomy. Acta Obstet Gynecol Scand 79:110-1104

16. McIntosh C, Dexter F, Epstein RH (2006) The impact of servicespecific staffing, case scheduling, turnovers, and first-case starts on anesthesia group and operating room productivity: a tutorial using data from an Australian hospital. Anesth Analg 103:14991516

17. Pandit JJ, Carey A (2006) Estimating the duration of common elective operations: implications for operating list management. Anaesthesia 61:768-776

18. Mirhashemi R, Harlow BL, Ginsburg ES, Signorello LB, Berkowitz R, Feldman S (1998) Predicting risk of complications with gynecologic laparoscopic surgery. Obstet Gynecol 92:327331

19. Myers ER, Steege JF (1999) Risk adjustment for complications of hysterectomy: limitations of routinely collected administrative data. Am J Obstet Gynecol 181:567-575

20. Dean MM, Finan MA, Kline RC (2001) Predictors of complications and hospital stay in gynecologic cancer surgery. Obstet Gynecol 97:721-724

21. Larsson PG, Platz-Christensen JJ, Forsum U, Pahlson C (1991) Clue cells in predicting infections after abdominal hysterectomy. Obstet Gynecol 77:450-452

22. Soper DE, Bump RC, Hurt WG (1990) Bacterial vaginosis and trichomoniasis vaginitis are risk factors for cuff cellulitis after abdominal hysterectomy. Am J Obstet Gynecol 163:1016-1021 